\section{MS29-P8 Quantitative estimate of cohesion forces}

Michał Kaźmierczak ${ }^{1}$, Andrzej Katrusiak ${ }^{1}$

1. Adam Mickiewicz University in Poznań

email: kax@amu.edu.pl

Distance between van der Waals spheres is a handy variable for description of intermolecular contacts. ${ }^{1-3}$ Its value vary between types of contacts but also within it. The idea behind presented research was to investigate the distribution of first, second and next shortest contacts with taking under consideration the type of contact. Changes of these distributions reflect role of specific types of interactions in the crystal structure (Figure 1). This information is extremely useful in crystal engineering and drug design because it sheds light on processes of molecular aggregation and phase transition. ${ }^{4}$

To describe investigated contacts two parameters were introduced: contact parameter $\delta$ (distance between van der Waals spheres) and contact order $n$ (position on list sorted by ascending value of contact parameter $\delta$ ). The survey was conducted on structural models from Cambridge Structural Database. ${ }^{5}$ For every investigated deposit ten shortest contacts were statistically analysed in terms of contact parameter $\delta$ and contact order $n$. Obtained distributions were approximate by split Pearson VII function.

Tabulated data obtained by fitting model function allows to compare various types of intermolecular interactions. Distributions of contact parameter $\delta$ as function of contact order $n$ show also very important role of strongest intermolecular interaction during processes of molecular aggregation and phase transition.

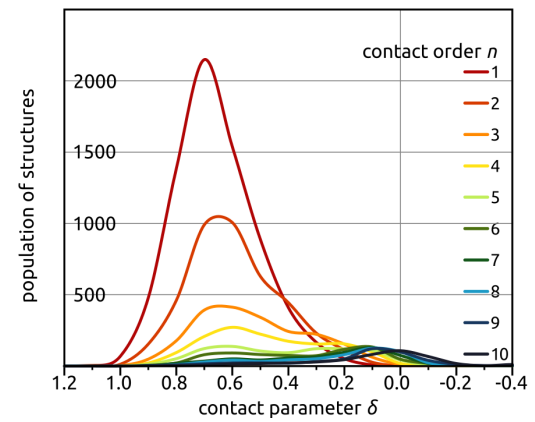

Figure 1. Distribution of contact $\mathrm{NH} \cdots \mathrm{N}$ in function of contact parameter $\delta$ for 10 first contact orders $n$. The bin-width $0.1 \AA$ was applied.

Keywords: intermolecular interactions, molecular assemblies, phase transition

\section{MS29-P9 Force field prediction of a reversal} transition in molecular crystals

\author{
Khadidja Brahimi $^{1}$, Juerg Hulliger ${ }^{1}$
}

1. Department of Chemistry and Biochemistry, University of Berne email: khadidjab7@gmail.com

Polarity formation is a general growth feature of systems showing unidirectional self-assembly of polar building blocks into a bulk state [1]. Channel-type inclusion compounds, single component molecular crystals, solid solutions, optically anomalous crystals, inorganic ionic crystals, biological tissues and biomimetic composites investigated experimentally all showed domains of opposite polarities in their final grown state [2]. In this field, molecular crystals made of dipolar organic molecules growing into a polar crystals structure are subject to undergo a reversal transition and open up fascinating opportunities for computational and experimental studies. In the frame of a general theory on stochastic polarity formation, the analytical description demonstrates that the reversal will take place in that direction (sectors involving the polar axis) where $180^{\circ}$ orientational defect formation is less endothermic [3]. Therefore, force field calculations of the energy of $180^{\circ}$ defect formation at both types of faces, i.e $(h k l)(h, k, l=$ $+1,0,-1)$ may allow to predict which side of the polar axis should show reversal of most of the dipoles. This basic property of molecular crystals is exemplified by investigating real systems for which at first we describe the structures and the representative $(h k l),(h, k, l=+1,0$ -1) faces for which interaction energies are explicitly calculated by taking into account the specific symmetry of the lattice. Conceptually a quite simple procedure, the practical elaboration can be rather complicated, because of the presence of different sites which are symmetry independent at the surfaces of different $(h k l)$ faces. The two chosen dipolar molecules are 1-chloro-4-nitrobenzonitrile and 1-bromo-4-cyanobenzonitrile. The molecules were selected as such to provide geometries and sizes which do not impose a significant surface site reconstruction (relaxation), when an $180^{\circ}$ reversed attachment is considered. We will present the results of a structural and energetical analysis involving different faces where we may define a difference of energy when docking a molecule down or up, by looking at the values of this energy difference predictive for the polar behaviour of individual faces. This configurational study highlights a relationship between the symmetry of a surface and the reversal transition which may occur when growth proceeds.

Keywords: Polar molecular crystals, Polarity, crystal growth 